\title{
The Advantage of Aerosol Manipulation by Twin Types of Acoustic Resonances
}

\author{
Zhenghui Qiao, Yaji Huang, Vincenzo Naso, and Wei Dong
}

\begin{abstract}
In order to make full use of the potential advantage of the multiple type of acoustic resonance, twin types of acoustic resonances (TTAR) are proposed to construct acoustic resonant device for manipulating aerosols with low energy consumption for acoustic source. The TTAR is realized by the frequency coupling between the Helmholtz resonator and the harmonic waveguide. The TTAR consists of the $1^{\text {st }}$ type of resonance based on the Helmholtz resonator and the $2^{\text {nd }}$ type of resonance based on the harmonic waveguide. The sound pressure generated at resonance frequency $1268 \mathrm{~Hz}$ caused by the 1st type of resonance is amplified 1.8 times comparing to that caused by the acoustic source without the 1st type of resonance. The peak sound pressure caused by TTAR is amplified 1.1 times than that caused by the single type of acoustic resonance based on the harmonic waveguide. The variation of sound pressure distribution for the TTAR and the STAR has the same regulation; however, the former peak sound pressure is much larger than the later at the same energy consumption for acoustic source. Especially, benefit from this advantage, the aerosols and the air medium in waveguide are separated by TTAR in the finite space within waveguide.
\end{abstract}

Index Terms $-\mathbf{P M}_{2.5}$, manipulation, acoustic wave, resonance, energy.

\section{INTRODUCTION}

The aerosol manipulation from the exhaust, such as biomass-fired smoke, coal-fired flue gas, diesel exhaust fumes, etc., plays the significant role in the aerosol removal for ensuring high air quality [1]. The aerosols contained heavy metals suspended in air can cause seriously healthy problem once they are inhaled by the respiratory system [2]. The study on different aerosol manipulation methods is an unnecessary aspect related to preserve air quality and human being healthy from aerosol pollution.

Recently, the aerosol manipulation by acoustic wave is considered as a potential aerosols removal method [3]. Many important studies have been carried out about acoustic aggregation [4]-[7], acoustic manipulation [8]-[10] and

Manuscript received October 14, 2015; revised February 2, 2016. This work was supported by the Fundamental Research Funds for the Central Universities of China under Grant 2242015k10023, the Scientific Research Foundation of Graduate School of Southeast University under Grant YBJJ1547, the National Nature Science Foundation of China under Grant 11190015 and the Research Innovation Program for College Graduates of Jiangsu Province of China under Grant KYLX15_0069.

Zhenghui Qiao and Yaji Huang are with the School of Energy \& Environment, Southeast University, Nanjing 210096, PR China (e-mail: seuqzh@seu.edu.cn, heyyj@seu.edu.cn).

Vincenzo Naso is with the Department of Mechanical and Aerospace Engineering, Sapienza University of Rome, Rome, Italy (e-mail: vincenzo.naso@gmail.com).

Wei Dong is with the School of Energy \& Environment, Southeast University, Nanjing 210096, PR China, he is also with the Department of Mechanical and Aerospace Engineering, Sapienza University of Rome, Rome, Italy (e-mail: dongwei59@ seu.edu.cn). acoustic levitation [11]-[14]. For example, Chen etc. [7] studied experimentally the diesel exhaust aerosols of size 0.023-10 $\mu \mathrm{m}$ can be obviously aggregated in one-dimension acoustic standing wave field of sound pressure $161.5 \mathrm{~dB}$ and single frequency $1 \mathrm{kHz}$. Karpul etc. [8] studied theoretically the industrial aerosols of radius $1 \mu \mathrm{m}$ can be separated from air in one-dimension acoustic standing wave field with consuming numerous acoustic energy. Boullosa etc. [14] experimentally levitated the polystyrene spheres of diameter $2 \mathrm{~cm}$ at sound pressure $150 \mathrm{~dB}$ using their acoustic device based on the Helmholtz resonator. In these studies, the high sound pressure generated at one appropriate frequency is one indispensable [15] condition for the implementation of these aerosol manipulation methods. However, for the current studies [4]-[7], [15], the production of high sound pressure generally consumes a large number of the energy from acoustic source. In terms of the numerous industrial aerosols emission [3], the numerous energy consumption of acoustic source becomes one significant impediment on the industrial implementation for these aerosol manipulation methods [4]-[8]. Therefore, in this paper, the aerosol manipulation with low energy consumption of acoustic source is an important study aspect of the aerosol manipulation by acoustic wave.

In the current studies [7]-[9], [11], [15], the acoustic resonance design of aerosol manipulation device is generally used to generate high sound pressure without high energy consumption based on the impendence matching characteristic [15] between device and air. The corresponding resonance type generally focuses on the unique one-dimension harmonic waveguide [7], [11], [15]. However, the number of acoustic resonance type is relatively fewer considered in the general harmonic mechanism of the unique waveguide. This acoustic resonance type can be named as single type of acoustic resonance (STAR).

In terms of the type amount of the resonance mechanism, we consider that the multiple types of acoustic resonances might contribute to reduce the energy consumption when generating high sound pressure. The coupling of several acoustic resonance types might be an important aspect for the progress of aerosol manipulation device with low energy consumption.

Base on our previous study [9], [10], we propose a new twin type of acoustic resonances (TTAR) with low energy consumption to be used to aerosol manipulation. It is well known that the waveguide has dual kinds of function, one for constructing acoustic field and the other for containing and manipulating aerosols. The operation mechanism of TTAR considered as the coupling of the $1^{\text {st }}$ type and the $2^{\text {nd }}$ type of resonance is used to construct the acoustic manipulation 
device of TTAR. The sound pressure characteristic deriving from the improvement of TTAR is studied. The realization of aerosol manipulation by TTAR would experimentally also be carried out.

\section{EXPERIMENT AND METHOD}

\section{A. Experimental Set-up and Mechanism}

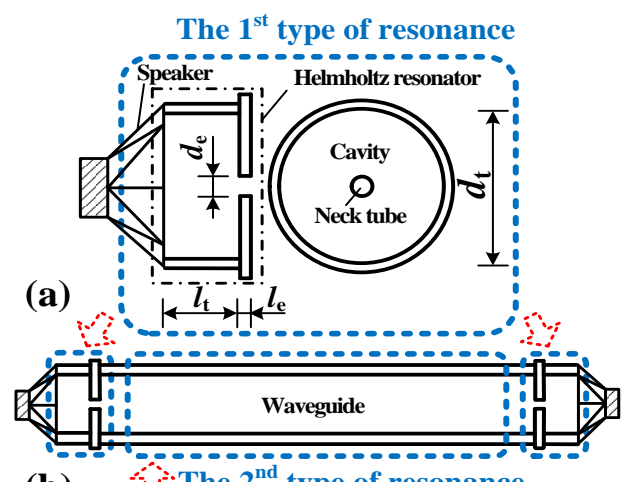

(b)

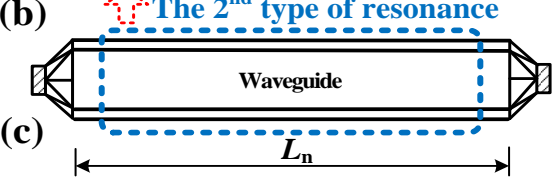

Fig. 1. Diagram of experimental set-up.

Fig. 1 shows the set-up diagram of TTAR. The set-up (b) corresponding to TTAR consists of the $1^{\text {st }}$ type of resonance component (a) based on Helmholtz resonator (HR) and the $2^{\text {nd }}$ type of resonance component (c) based on one-dimension harmonic waveguide. The geometric parameters of set-up components are showed in Fig. 1.

The resonance principle of the $1^{\text {st }}$ type of resonance depends on relation formula Eq. (1).

$$
f^{2}=\left(c^{2} /\left(4 \pi^{2}\right)\right)\left(\pi d_{\mathrm{e}}^{2} / 4\right) /\left(l_{\mathrm{e}}+0.73 d_{\mathrm{e}}\right) /\left(l_{\mathrm{t}} \pi d_{\mathrm{t}}^{2} / 4\right)
$$

where $f$ is the Helmholtz resonance frequency of HR; $c$ is the acoustic velocity of air; $d_{\mathrm{e}}, l_{\mathrm{e}}$ and $d_{\mathrm{t}}, l_{\mathrm{t}}$ are the geometric parameters of HR.

The resonance principle of the $2^{\text {nd }}$ type of resonance depends on relation formula Eq. (2).

$$
f_{n}=n c /(2 L)
$$

where $f_{n}$ is the $n$-th order resonance frequency of harmonic waveguide; $n$ is the harmonic order of waveguide.

In the TTAR system, the basically coupling between the two resonance types depends on the numeric value equivalency for the two resonance frequencies in Eq. (1) and Eq. (2). This coupled equivalency is the key operation mechanism between the single and twin resonance types. Note that the realization of traditional acoustic resonance [7], [8], the so-called STAR, depends simply on the resonance principle represented as Eq. (2). In the device study for the STAR, two opposing traditional acoustic sources such as the speakers are generally arranged at the two ends of waveguide showed in Fig. 1(c). This acoustic device does not consider the resonance principle represented as Eq. (1). In this work, we utilize the characteristic comparison between the STAR and the TTAR to verify the advance performance of TTAR. In order to demonstrate the performance of TTAR, the experimental parameters of set-up are respectively $L=$ $978 \mathrm{~mm}, n=7, d_{\mathrm{e}}=8 \mathrm{~mm}, l_{\mathrm{e}}=5 \mathrm{~mm}, d_{\mathrm{t}}=35 \mathrm{~mm}, l_{\mathrm{t}}=9 \mathrm{~mm}$ and $f$ $=f_{n=7}=1268 \mathrm{~Hz}$.

\section{B. Method}

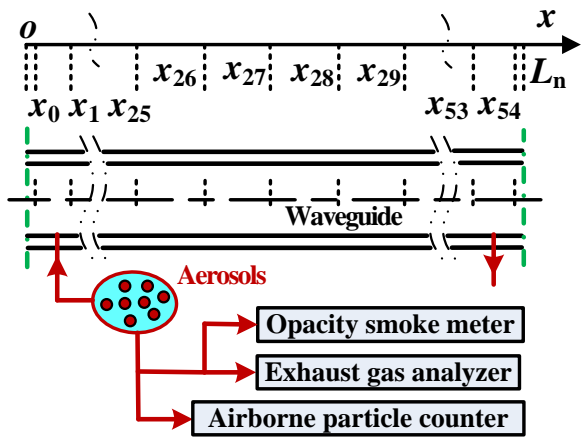

Fig. 2. Experiment diagram.

Considering the dual kinds of functions of waveguide, Fig. 2 shows the experiment measurement of the $2^{\text {nd }}$ type of resonance compound on aerosol manipulation. This measurement in waveguide is always appropriate no matter for testing the TTAR or for testing the STAR. The arrangement positions for testing sound pressure within harmonic waveguide and the aerosol characteristic measurement are showed in Fig. 2. $x_{3}, x_{11}, x_{19}, x_{27}, x_{35}, x_{43} \&$ $x_{51}$ and $x_{7}, x_{15}, x_{23}, x_{31}, x_{39} \& x_{47}$ correspond to the ant-nodes and nodes of acoustic field. $x_{1}, x_{5}, x_{9}, x_{13}, x_{17}, x_{21}, x_{25}, x_{29}, x_{33}$, $x_{37}, x_{41}, x_{45}, x_{49}$ and $x_{53}$ correspond to the middles between adjacent nodes and anti-nodes. $x_{2}, x_{4}, x_{10}, x_{12}, x_{18}, x_{20}, x_{26}, x_{28}$, $x_{34}, x_{36}, x_{42}, x_{44}, x_{50} \& x_{52}$ and $x_{6}, x_{8}, x_{14}, x_{16}, x_{22}, x_{24}, x_{30}, x_{32}, x_{38}$, $x_{40}, x_{46} \& x_{48}$ correspond to the positions to be away from nodes and antinodes of $15 \mathrm{~mm}$. The source of aerosol material is tobacco fired smoke of aerosol size distribution containing $\leq 0.3 \mu \mathrm{m}$ of $69 \%$ aerosols, $0.3 \mu \mathrm{m}-0.5 \mu \mathrm{m}$ of $28 \%$ aerosols and $>0.5 \mu \mathrm{m}$ of $3 \%$ aerosols measured by airborne particle counter (Aero Track APC 9303-01). The aerosol number concentration is larger than $50 \%$ measured by opacity smoke meter (SV-5Y). The gas compounds consist of $\mathrm{NO}_{\mathrm{x}}(54.5 \mathrm{ppm})$, $\mathrm{CO}(1.43 \%), \mathrm{CO}_{2}(3.95 \%), \mathrm{O}_{2}(18.95 \%)$ measured by exhaust gas analyzer $(\mathrm{SV}-5 \mathrm{Q})$. The experiment temperature is $25^{\circ} \mathrm{C}$.

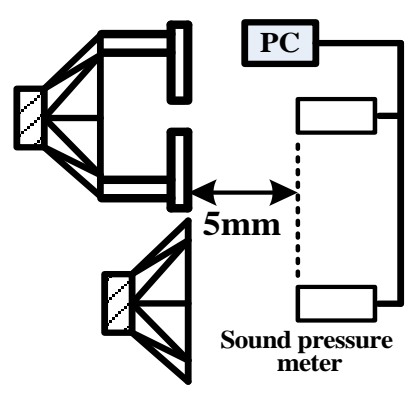

Fig. 3. The $1^{\text {st }}$ type resonance test.

Fig. 3 shows the test method for the $1^{\text {st }}$ type of resonance compared to the general acoustic source. The distances between the acoustic source and sound pressure meter are the same. Note that with the distance increase, the resonance responds have the similar variation trend. Here, we only focus on the variation for the distance equal to $5 \mathrm{~mm}$. 


\section{RESULTS AND ANALYSIS}

A. The Characteristic of TTAR
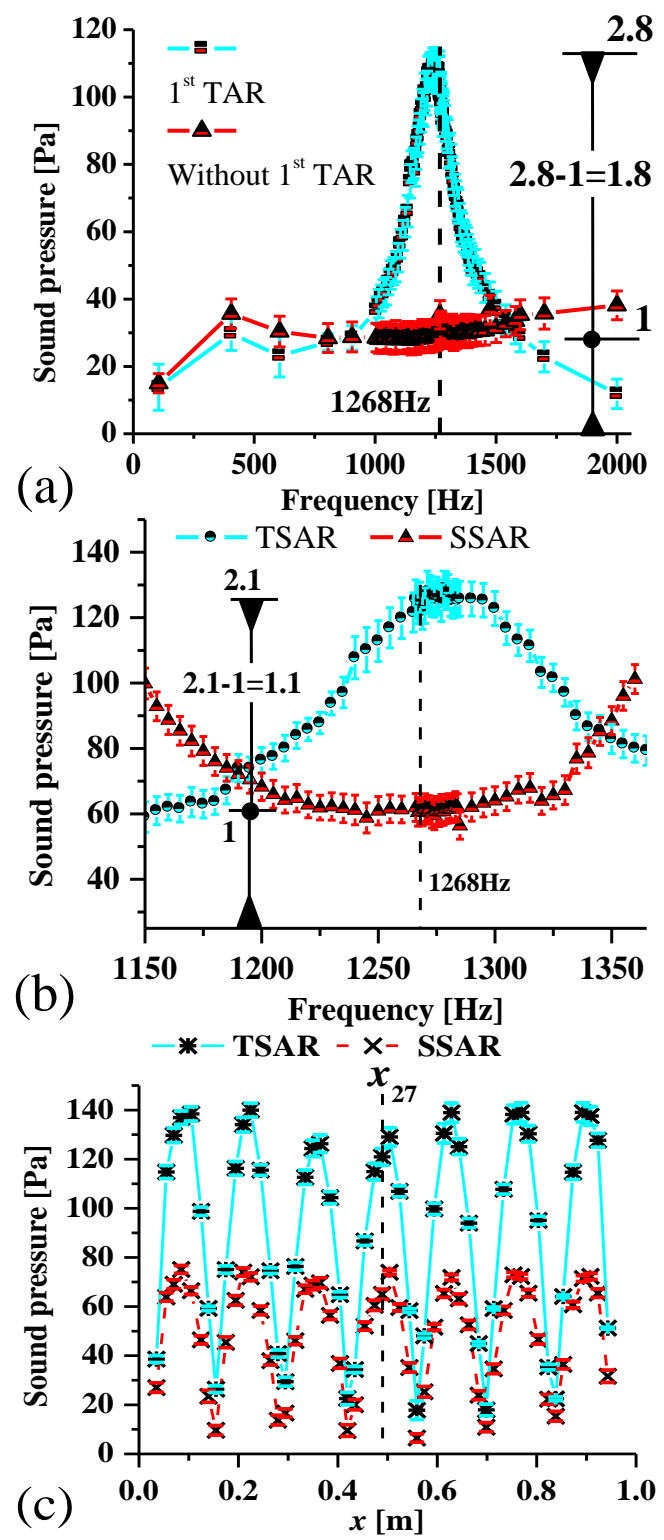

Fig. 4. The comparison characteristic.

In Fig. 4, (a) is the comparison of the $1^{\text {st }}$ type of acoustic resonance (represented as $1^{\text {st }}$ TAR) to the general acoustic source (represented as without $1^{\text {st }} \mathrm{TAR}$ ); (b) is the comparison of the TTAR to the STAR; (c) is the comparison of acoustic fields respectively caused by the TTAR and the STAR. Note that the energy consumption of acoustic source for the corresponding comparison experiments is same.

Fig. 4 shows the advance acoustic characteristic of TTAR. In terms of the $1^{\text {st }}$ type of acoustic resonance showed in Fig. 4(a), the resonance respond curve on sound pressure vs. frequency has an obvious peak value at resonance frequency near to the design frequency of acoustic device. This phenomenon indicates that the resonance principle of the $1^{\text {st }}$ type of acoustic resonance can cause larger sound pressure compared to the general acoustic source. The sound pressure is amplified 1.8 times at the designed frequency of $1268 \mathrm{~Hz}$. In terms of TTAR showed in Fig. 4(b), the resonance resounds with a peak value is also caused at the frequency range of center frequency near $1268 \mathrm{~Hz}$. The sound pressure caused by
TTAR increases 1.1 times than that caused by STAR at $1268 \mathrm{~Hz}$. Fig. 4(c) shows the sound pressure distribution constructed in waveguide respectively by the TTAR and the STAR. The two resonance systems cause the obvious difference between the numerical values of the peaks, despite the similar seven peaks of sound pressure distribution. The spatial periodicity of the sound pressure distribution demonstrates the generation of acoustic standing wave (ASW) field within waveguide. For the sound pressure distribution in each resonance system, each peak has similar numeric value. The similar sound pressure variations indicate the realization of action effect caused by the $2^{\text {nd }}$ type of resonance principle for the two acoustic devices corresponding to TTAR and STAR. The obviously increased sound pressure for TTAR indicates the advantage of using TTAR to generate high sound pressure without much energy consumption for acoustic source.

\section{B. The Aerosol Manipulation by TTAR}

In Fig. 5, (a) represents the initial aerosol distribution in waveguide; (b) represents the aerosol distribution caused by the TTAR for the sustaining 8 minutes and 26 seconds of acoustic wave; (c) represents the aerosol distribution caused by the STAR with sustaining same action time of acoustic wave. For the two acoustic devices, the energy consumptions for acoustic source during the two comparisons are the same $6.74 \mathrm{~W}$. The peak sound pressure caused by TTAR is $126 \mathrm{~Pa}$ at $1268 \mathrm{~Hz}$. The bright region and the dark region in waveguide represent the distribution region of aerosols within waveguide. The bright region corresponds to the high number concentration of aerosols. The dark region corresponds to the low number concentration of aerosols. The triangle region $A$ represents the region of low aerosol concentration. The triangle regions $B_{\mathrm{I}}$ and $B_{\mathrm{II}}$ represent the two similar regions of high aerosol concentration. $\mathrm{AN}$ and $\mathrm{N}$ represents the anti-node and node of ASW field. $\lambda$ represents the wavelength of acoustic wave.

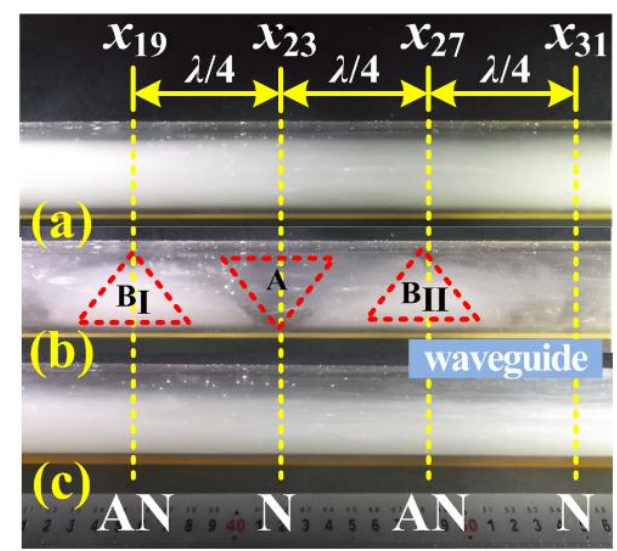

Fig. 5. Aerosol manipulation effect.

Fig. 5 shows the advance characteristic of aerosol manipulation by TTAR. Corresponding to the dual kinds of functions of waveguide, the realization of aerosol manipulation by TTAR consists of two processes, the generation of ASW field by TTAR and the aerosol manipulation in ASW field. Fig. 3(c) indicates the productions of ASW field by TTAR and STAR. In ASW field, the mechanism of aerosol manipulation by acoustic wave can 
refer to [8], [9], [16], [17]. Due to the acoustic radiation force exerting on aerosols in waveguide, the aerosols in the regions closed to the nodes of ASW field are driven to the regions closed to the contiguous anti-nodes. Based on the mass conservation, the air medium in waveguide is accumulated to the regions near the nodes. At the initial action moment of acoustic wave, the aerosol number distribution in waveguide is uniformly zonal showed in Fig. 4(a). In ASW field, after 8 minutes and 26 seconds, two types of accumulation regions are caused due to the directional driven for aerosols and the sustaining accumulation for air medium between nodes and anti-nodes. The two types of regions correspond to aerosol accumulation region, $B_{\mathrm{I}}$ and $B_{\mathrm{II}}$, and air accumulation region, $A$, showed in Fig. 4(b). Fig. 4(c) shows that after same manipulation time, the aerosol number distribution caused by STAR is still zonal. The manipulation effect caused by STAR similar to that caused by TTAR does not appear. The reason might be the low [8] sound pressure caused by STAR with the same energy consumption in TTAR. Considering that one practical passageway can be constructed at the aerosol or air accumulation region, the high concentration aerosols or the more pure air can be gained. Therefore, the TTAR might be appropriate to the industrial aerosol removal.

\section{CONCLUSION}

The TTAR focusing on the number of acoustic resonance types is successfully used to manipulate aerosols. Different from the traditional STAR depending on the sole acoustic resonance principle based on the unique harmonic waveguide, the TTAR depends on two different acoustic resonance principles respectively based on the Helmholtz resonator and harmonic waveguide. The two resonance principles are named as the $1^{\text {st }}$ type of resonance and the $2^{\text {nd }}$ type of resonance. The two different resonances are coupled by the resonance frequency equivalency. It was found that under the same energy consumption for acoustic source, the sound pressure peak value of resonance responds caused by TTAR is 2.1 times than the sound pressure caused by STAR.In terms of the $1^{\text {st }}$ type of resonance, the sound pressure peak value of resonance responds is 2.8 times than the sound pressure caused by the acoustic source without considering the $1^{\text {st }}$ type of resonance. The resonance peaks occur at the center frequency near $1268 \mathrm{~Hz}$. Based on the same variation periodicity of sound pressure distribution for TTAR and STAR, the sound pressure caused by TTAR is much larger than STAR. Comparing to the aerosol manipulation by STAR the aerosol accumulation region and air accumulation region reveal the more effective manipulation effect by TTAR.

The experimental aerosol source material is tobacco fired smoke. The aerosol size distribution are $\leq 0.3 \mu \mathrm{m}(69 \%$ aerosols $), 0.3 \mu \mathrm{m}-0.5 \mu \mathrm{m}$ ( $28 \%$ aerosols $)$ and $>0.5 \mu \mathrm{m}(3 \%$ aerosols).

\section{REFERENCES}

[1] D. Y. H. Pui, S. Chen, and Z. Zuo, "PM2.5 in China: Measurements, sources, visibility and health effects, and mitigation," Particuology, vol. 13, pp. 1-26, 2014.

[2] P. H. Brunner and H. Rechberger, "Waste to energy — key element for sustainable waste management," Waste Manage, vol. 37, pp. 3-12, 2015.
[3] Q. Yao, S. Q. Li, H. W. Xu, J. K. Zhuo, and Q. Song, "Reprint of: studies on formation and control of combustion particulate matter in China: A review, " Energy, vol. 35, pp. 4480-4493, 2010.

[4] Q. J. Guo, Z. N. Yang, and J. S. Zhang, "Influence of a combined external field on the agglomeration of inhalable particles from a coal combustion plant," Powder Techno, vol. 227, pp. 67-73, 2012.

[5] H. Chen, Z. Luo, J. Jiang, D. Zhou, M. Lu, M. Fang et al., "Effects of simultaneous acoustic and electric fields on removal of fine particles emitted from coal combustion," Powder Techno., vol. 281, pp. 12-19, 2015.

[6] J. Z. Liu, G. X. Zhang, J. H. Zhou, J. Wang, W. D. Zhao, and K. F. Cen, "Experimental study of acoustic agglomeration of coal-fired fly ash particles at low frequencies," Powder Technol., vol. 193, pp. 20-25, 2009.

[7] H. Chen, R. Zhang, J. Cao, and X. Shen, "Experimental study on acoustic agglomeration of ultrafine particles in diesel engine exhaust, " Neiranji Xuebao/Transactions of CSICE (Chinese Society for Internal Combustion Engines), vol. 27, pp. 160-165, 2009.

[8] D. Karpul, J. Tapson, M. Rapson, A. Jongens, and G. Cohen, "Limiting factors in acoustic separation of carbon particles in air," J. Acoust. Soc. Am., vol. 127, pp. 2153-2158, 2010.

[9] Z. Qiao, H. Yaji, N. Vincenzo, and W. Dong, "Aerosol manipulation by acoustic tunable phase-control at resonant frequency," Powder Technol., vol. 281, pp. 76-82, 2015.

[10] Z. Qiao, Y. Huang, and W. Dong, "Acoustic resonance characteristics of symmetric cylindrical waveguide with Helmholtz sound source," Journal of Southeast University (Natural Science Edition), vol. 44, pp. 579-584, 2014.

[11] D. Foresti, G. Sambatakakis, S. Bottan, and D. Poulikakos, "Morphing surfaces enable acoustophoretic contactless transport of Ultrahigh-Density matter in air," Sci. Rep.-UK., vol. 3, p. 3176, 2013.

[12] M. červenka and M. Bednařík, "Acoustic particle displacement resonator," Appl. Acoust., vol. 99, pp. 155-160, 2015.

[13] J. K. R. Weber, C. A. Rey, J. Neuefeind, and C. J. Benmore, “Acoustic levitator for structure measurements on low temperature liquid droplets," Rev. Sci. Instrum., vol. 80, p. 083904, 2009.

[14] R. R. Boullosa and F. Orduna-Bustamante, "Acoustic levitation at very low frequencies," Acta Acust. Acust., vol. 96, pp. 376-382, 2010.

[15] J. A. Gallego-Juarez, E. De Sarabia, G. Rodriguez-Corral, T. L. Hoffmann, J. C. Galvez-Moraleda, J. J. Rodriguez-Maroto et al., "Application of acoustic agglomeration to reduce fine particle emissions from coal combustion plants," Environ. Sci. Techno., vol. 33, pp. 3843-3849, 1999.

[16] L. Gor'Kov, "On the forces acting on a small particle in an acoustical field in an ideal fluid," Sov. Phys. Dokl., vol. 6, pp. 773-775, 1962.

[17] J. Greenhall, F. G. Vasquez, and B. Raeymaekers, "Continuous and unconstrained manipulation of micro-particles using phase-control of bulk acoustic waves," Appl. Phys. Lett., vol. 103, p. 074103, 2013.

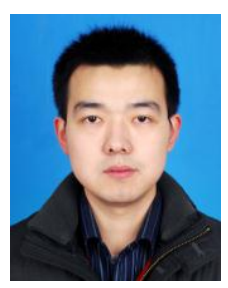

Zhenghui Qiao was born in Nanyang, Henan province of China, in 1988. He received the bachelor's degree of architectural environment \& equipment engineering (HVAC) from Zhongyuan University of Technology, Zhengzhou, PR China in 2011. He received the master's degree of power engineering specialty in Southeast University, Nanjing, PR China in 2013.

He is now a PhD student of the School of Energy \& Environment, Southeast University, Nanjing, PR China. His major field of study is power engineering and thermal physics. Recently, he published a journal article in Powder Technology (Switzerland, Elsevier Science BV, 2015). The title of the article is "Aerosol manipulation by acoustic tunable phase-control at resonant frequency." The current and previous research interests include renewable energy utilization technology, recycling and utilization of environmental energy, micro energy conversion and utilization, $\mathrm{PM}_{2.5}$ emission control using the energy, the aerosol manipulation from in air using acoustic standing wave, and the acoustic field construction for the manipulation.

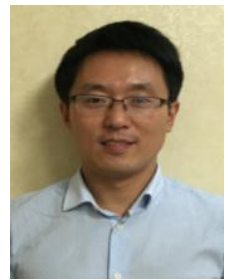

Yaji Huang was born in Rugao, Jiangsu province of China, in 1975. He received the doctor's degree of power engineering and thermal physics in Southeast University, Nanjing, China in 2004.

Now, he is the vice president and a doctoral tutor of the School of Energy and Environment, Southeast University.

$\mathrm{He}$ was an associate professor of the School of 
Energy and Environment in Southeast University, from 2005-2012. He has been a professor of School of Energy and Environment in Southeast University since 2012.

He has published more than 50 scientific papers at international or national levels (journals, proceedings of international conferences). The major field of study contains clean coal utilization technology and renewable energy utilization technology.

Prof. Huang is selected for the new century talent program of the Ministry of Education of PR China, the Jiangsu province "Blue Project" outstanding young teachers, and the outstanding young teacher training program of Southeast University. He won the first prize of science and technology progress in Jiangsu province of PR China for once. He won the second prize of science and technology progress in the Ministry of Education and Jiangsu province of PR China for three times.

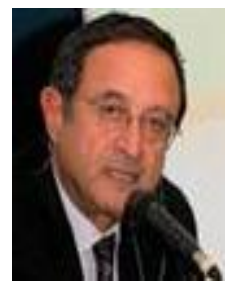

Vincenzo Naso was born in Naples of Italy in 1944 He received his $\mathrm{PhD}$ (2 years) degree at the School of Aeronautics and Aerospace Engineering, Rome University "la Sapienza" in 1971.

Now, he is a professor of Aeronautics and Aerospace Engineering Department at Rome University "la Sapienza".

He had been an assistant professor of energy conversion systems at Rome University "la Sapienza", from 1971 to 1973 . He was an associate professor of energy conversion systems at Ancona University, from 1972 to 1976. Then, he was an associate professor of energy conversion systems at Rome University "la Sapienza", from 1976 to 1982. From 1982 to 1986, he had been an associate professor of special energy conversion systems at Rome University "la Sapienza".

From 1986, he had been a full professor of engines and special energy conversion systems at Rome University "la Sapienza". From 1988 to 1990, he was a temporary professor of industrial plants at Rome University "la Sapienza”. Since 1992, he was a professor of energy saving in industrial systems at the master course on industrial management, Rome University "la Sapienza". Then he became a professor of energy cycle and its environmental impact at the master course on technical operator for developing countries, Rome University "la Sapienza", from 1999.

$\mathrm{He}$ has published more than 200 scientific papers at international or national levels (journals, proceedings of international conferences, workshops), two books on innovative energy conversion systems and clean technologies (in Italian), one book on stirling engines (both in Italian and in English, Milan, 2008) as well as one on energy system in era of energy vectors (in English, Springer, 2010).

The major fields of his study is energy conversion systems including: energy conversion systems; new and renewable energy conversion systems; clean energy vectors (hydrogen); energy saving in industry, transport and other end uses; clean technologies for sustainable development; sustainability science; environmental, social and esychological impact of energy systems; international co-operation and technology transfer and innovation.

Prof. Naso is a member of American Society of Mechanical Engineering (ASME), USA; Associazione di Tecnica dell'Automobile (A.T.A.), Italia Associazione di Tecnica Navale (A.TE.NA.), Italia; and Associazione Termotecnica Italiana (A.T.I.), Italia, etc.

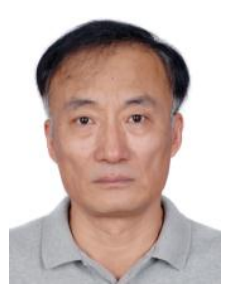

Wei Dong was born in Xian, Shannxi province of China in 1959. He received the $\mathrm{PhD}$ degree (energetic engineering) from the Energetic Engineering Department of Rome University "la Sapienza”, Rome, Italy in 1998.

Now, he is a professor with the School of Energy and Environment in Southeast University.

He was an assistant of the Mechanical Engineering Department of Tianjin TV University, from 1982-1990; then he became a lecturer of the Mechanical Engineering Department of Tianjin TV University, from 1990-1993. From 1998, he was a researcher of Aeronautics and Aerospace Engineering Department, at Rome University "la Sapienza". Since 1998, he was an associated member of CIRPS - Interuniversity Research Center on Sustainable Development of Rome University "La Sapienza", and he became a professor of School of Energy and Environment in Southeast University in 2002.

$\mathrm{He}$ has published more than 50 scientific papers at international or national levels (journals, proceedings of international conferences).

The major fields of his study are conventional and advanced energy transfer and conversion systems including micro energy transfer and conversion system and advanced technologies in harvesting energy from environment 
Electrical Energy 
